J. Dairy Sci. 99:1619-1631

http://dx.doi.org/10.3168/jds.2015-10253

(C) American Dairy Science Association ${ }^{\circledR}, 2016$.

\title{
Field validation of protocols developed to evaluate in-line mastitis detection systems
}

\author{
C. Kamphuis, ${ }^{1,2}$ B. T. Dela Rue, and C. R. Eastwood \\ DairyNZ Ltd., Hamilton 3240, New Zealand
}

\section{ABSTRACT}

This paper reports on a field validation of previously developed protocols for evaluating the performance of in-line mastitis-detection systems. The protocols outlined 2 requirements of these systems: (1) to detect cows with clinical mastitis $(\mathrm{CM})$ promptly and accurately to enable timely and appropriate treatment and (2) to identify cows with high somatic cell count (SCC) to manage bulk milk SCC levels. Gold standard measures, evaluation tests, performance measures, and performance targets were proposed. The current study validated the protocols on commercial dairy farms with automated in-line mastitis-detection systems using both electrical conductivity (EC) and SCC sensor systems that both monitor at whole-udder level. The protocol for requirement 1 was applied on 3 commercial farms. For requirement 2, the protocol was applied on 6 farms; 3 of them had low bulk milk SCC $\left(128 \times 10^{3}\right.$ cells $\left./ \mathrm{mL}\right)$ and were the same farms as used for field evaluation of requirement 1 . Three farms with high bulk milk SCC $\left(270 \times 10^{3}\right.$ cells $\left./ \mathrm{mL}\right)$ were additionally enrolled. The field evaluation methodology and results were presented at a workshop including representation from 7 international suppliers of in-line mastitis-detection systems. Feedback was sought on the acceptance of standardized performance evaluation protocols and recommended refinements to the protocols. Although the methodology for requirement 1 was relatively labor intensive and required organizational skills over an extended period, no major issues were encountered during the field validation of both protocols. The validation, thus, proved the protocols to be practical. Also, no changes to the data collection process were recommended by the technology supplier representatives. However, 4 recommendations were made to refine the protocols: inclusion of an additional analysis that ignores small (low-density) clot observations in the definition of $\mathrm{CM}$, extension of the

Received August 14, 2015.

Accepted October 19, 2015.

${ }^{1}$ Corresponding author: claudia.kamphuis@wur.nl

${ }^{2}$ Current address: Chair Group Business Economics, Wageningen University, Hollandseweg 1, 6706KN, Wageningen, the Netherlands.

time window from 4 to 5 milkings for timely alerts for $\mathrm{CM}$, setting a maximum number of 10 milkings for the time window to detect a $\mathrm{CM}$ episode, and presentation of sensitivity for a larger range of false alerts per 1,000 milkings replacing minimum performance targets. The recommended refinements are discussed with suggested changes to the original protocols. The information presented is intended to inform further debate toward achieving international agreement on standard protocols to evaluate performance of in-line mastitisdetection systems.

Key words: automated mastitis detection, in-line sensors, performance evaluation, standard protocols

\section{INTRODUCTION}

Increasing herd sizes in major dairying countries is driving a need for automation and technologies that help farmers with their daily management decisions (Jago et al., 2013). In-line mastitis-detection systems have been developed to automate some tasks in management of mastitis and bulk milk SCC (BMSCC). Kamphuis et al. (2013a) reported the development of protocols to evaluate the performance of these systems against 2 criteria of importance to farmers. The systems need to identify cows with clinical mastitis (CM) and identify cows with a high SCC to manage BMSCC. Adoption rates of in-line mastitis-detection systems are reported to be $\sim 26 \%$ among surveyed dairy farmers internationally who have also invested in other sensor technologies (Borchers and Bewley, 2015). In the Netherlands, adoption rates of $35 \%$ with conventional milking systems and $93 \%$ with automatic milking systems have been reported (Steeneveld and Hogeveen, 2015) for mastitis-detection systems based on electrical conductivity (EC). Edwards et al. (2015) reported a much lower adoption rate of $6 \%$ for in-line mastitis-detection systems in a representative survey of New Zealand dairy farmers. Farmers without an in-line mastitis-detection system ranked such systems in the top 3 of potentially useful technologies for their farm (Borchers and Bewley, 2015) or expressed an interest in investing in them (Edwards et al., 2015). Reasons for dairy farmers not to invest in decision support technolo- 
gies include the perception that current commercially available technologies are unproven, unreliable, and have an uncertain return on investment (Russell and Bewley, 2013; Borchers and Bewley, 2015; Steeneveld and Hogeveen, 2015).

Several scientific publications have reported on inline mastitis-detection technologies, or new detection algorithms, which has been reviewed by Rutten et al. (2013). Most studies reported traditional performance indicators, such as sensitivity (Se; the proportion of gold standard-positive cases that are correctly detected as being positive for mastitis) and specificity (Sp; the proportion of gold standard-negative cases that are correctly detected as negative for mastitis). However, these performance indicators are difficult to interpret for nonscientific readers (Sherlock et al., 2008). Comparing performance indicators between studies, and thus between technologies and algorithms, is also extremely difficult because studies have used a variety of gold standards to define mastitis (Hogeveen et al., 2010). Claycomb et al. (2009) demonstrated that even small differences in gold standard definitions had a large effect on the number of true cases used for evaluation purposes. Additionally, whereas the gold standards between studies are similar, comparison of results remains difficult due to differences in the inclusion or exclusion of criteria used to create data sets to develop and validate detection models. For example, Kamphuis et al. (2010a) evaluated the performance of a mastitisdetection algorithm using 2 validation sets with differing certainty of mastitis status and found a difference in Se of $\sim 50 \%$. Last, studies use different time windows in which mastitis alerts are considered true positive or false negative. Kamphuis et al. (2010b) demonstrated that increasing the length of the time window significantly affects performance indicators: using a 24-h time window preceding the occurrence of a $\mathrm{CM}$ episode resulted in an Se of $40 \%$ at an Sp of $99 \%$. Increasing the time window to $96 \mathrm{~h}$ preceding the occurrence until 72 $\mathrm{h}$ after the occurrence of a CM episode increased Se to $75 \%$ at the same Sp level of $99 \%$.

To overcome the difficulties of interpreting performance indicators and the lack of uniform performance information, Kamphuis et al. (2013a) proposed a methodology to field evaluate the performance of in-line mastitis-detection systems, referred to as the protocols in the current paper, with respect to the 2 requirements (finding cows with $\mathrm{CM}$, and finding cows with high SCC to manage BMSCC levels). The protocols are aimed at providing (1) robust and uniform information on performance of current in-line mastitis-detection systems against criteria of importance to farmers to support more informed investment decisions, and (2) a performance evaluation framework to help technol- ogy suppliers develop or improve their technologies. The protocols were developed using literature, expert knowledge, and data from previous trials, but were not validated in their final form on commercial farms. This paper reports on the application of the protocols on commercial farms to evaluate their practicality and to refine the methodology using analysis of the field validation data and feedback from technology supplier representatives.

\section{MATERIALS AND METHODS}

In our study, the protocols for identifying cows with CM and identifying cows with high SCC to manage BMSCC were validated. As the protocols involve different data collection steps and statistical analysis, the materials and methods are described for each requirement separately.

\section{Requirement 1: Identifying Cows with CM Promptly and Accurately}

Data were collected as per the protocol from 3 commercial dairy farms, located in South Canterbury, New Zealand. The farms were selected from a list provided by a supplier of mastitis-detection systems. All farms milked large ( $>500$ cows), spring-calving herds through rotary parlors that had 2 in-line mastitis-detection systems installed. The systems recorded the maximum value of $\mathrm{EC}\left(\mathbf{E C}_{\max }\right)$ at every stall and $\mathrm{SCC}$ at every fourth stall where the SCC sensors were installed. Both sensor systems monitored whole-udder milk of individual cows. All 3 farms had mastitis-detection systems from the same supplier. Farm and data collection details are provided in Table 1. Before the trial started, a supplier representative conducted an inspection to confirm proper functioning of the sensor equipment.

The protocol recommends collection of a minimum of $20 \mathrm{CM}$ episodes per farm. As the incidence of CM is higher during early lactation, data were collected early in the milking season of 2012/2013 (Table 1). Data on clot observations for determination of $\mathrm{CM}$ episodes were collected as per the protocol for requirement 1. In-line filters with removable stainless steel screens (Vision Mastitis Detector; Ambic Equipment Ltd., Witney, UK) were fitted to the long milk tubes at every stall. These screens were removed and visually inspected by trained staff for the presence of clots (visually assessed as $>2 \mathrm{~mm}^{2}$ ) after each cow completed milking. On farm 1 clot observations were made by farm staff; external personnel were used on the other 2 farms. Both farm staff and external personnel were trained in the procedure of observing clots and recording data. When clots were identified, the filter screen was removed and replaced with a new one. The removed filter was placed 
Table 1. Herd and milking rotary size, clot observation period, total number of clot observations, total number of clot observations merged with milking data, and the total number of milkings during the clot observation period

\begin{tabular}{lcclccc}
\hline Farm & $\begin{array}{c}\text { Herd } \\
\text { size }\end{array}$ & $\begin{array}{c}\text { Milking } \\
\text { rotary size } \\
\text { (no. of stalls) }\end{array}$ & $\begin{array}{l}\text { Clot observation } \\
\text { period during 2012 }\end{array}$ & $\begin{array}{c}\text { Clot } \\
\text { observations } \\
\text { (no.) }\end{array}$ & $\begin{array}{c}\text { Clot } \\
\text { observations with } \\
\text { milking data (no.) }\end{array}$ & $\begin{array}{c}\text { Milkings during } \\
\text { clot observation } \\
\text { period (no.) }\end{array}$ \\
\hline 1 & 1,221 & 60 & October 10-4 & 282 & $246^{1}$ & 33,459 \\
2 & 538 & 50 & October 23-November 19 & 532 & $461^{2}$ & 27,328 \\
3 & 819 & 60 & November 20-December 12 & 296 & $147^{3}$ & 86,454 \\
Total & 2,578 & & & 1,110 & 854 & 87,241 \\
\hline
\end{tabular}

${ }^{1}$ There was 1 clot observation recorded twice but with different stall number and different density scores. Images were checked, and 1 clot observation was removed. Another 35 clot observations could not be matched with milking data.

${ }^{2}$ There was 1 clot observation recorded twice and 70 clot observations that could not be matched with milking data.

${ }^{3}$ There were 134 clot observations that had no milking data, another 15 clot observations had different stall numbers and could not be merged.

in a plastic filter housing and recorded with the cow identification number and stall number. Date and milking session (morning or evening) were recorded when each clot filter was photographed, enabling matching of clot observations with milking data (Figure 1). These images were later used to score the observed clots for their density (representing the percentage of area of the in-line filter covered) by a trained technician using scored clots in the protocol development study (Kamphuis et al., 2013a) as a training set.

Milking data collected from the herd-management software systems included cow identification number, stall number, date and time, milking session, and sensor measurements $\left(\mathrm{EC}_{\max }\right.$ and $\left.\mathrm{SCC}\right)$. Sensor measurements were not corrected for outlier values. Clot observations and milking data were merged by date, milking session, cow identification number, and stall number. A total of 854 clot observations and 87,241 milkings were used for further analysis (Table 1).

Clot observations were used to define CM episodes (Table 2): in the protocols a CM episode was defined as the observation of clots at 2 out of 3 or all 3 consecutive cow milkings. A CM episode ends at the last milking in which clots are observed followed by 4 consecutive cow milkings without clot observation. Milkings with a clot observation not belonging to a CM episode (that is, clots observed at 1 milking only) were excluded, as were sensor alerts at these excluded milkings and sensors alerts that appeared 1 milking before or after these excluded milkings. The remaining sensor alerts were used to calculate 2 sensitivities. For overall sensitivity $\left(\mathbf{S e}_{\text {overall }}\right)$, a sensor is expected to alert at least once

(a)

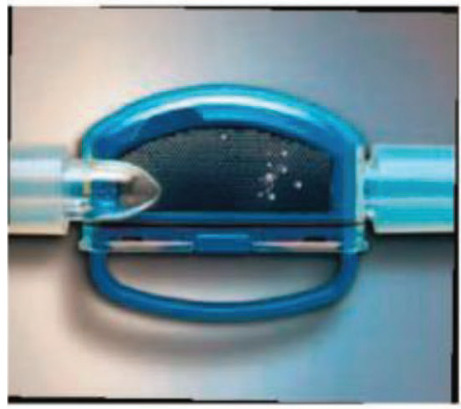

Low density

(b)

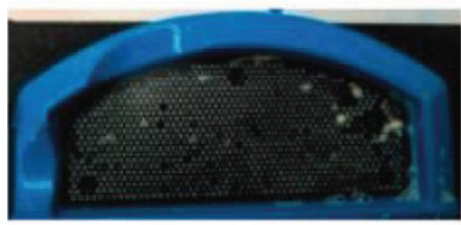

Low density

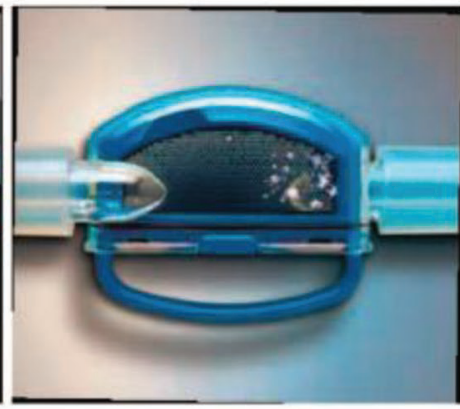

Medium density

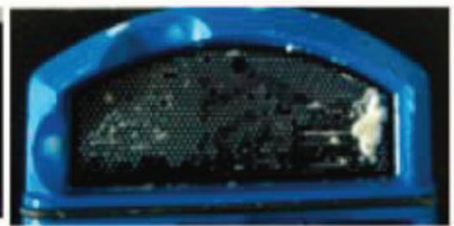

Medium density

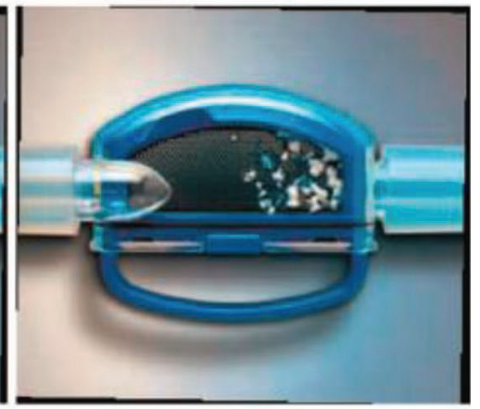

High density

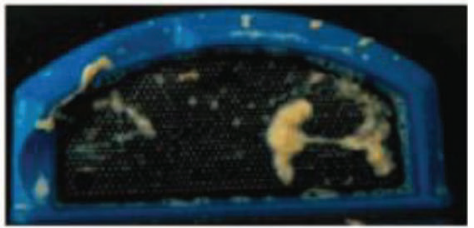

High density

Figure 1. (a) Clot density score guideline for clots observed on in-line filters (Ambic Equipment Ltd., Witney, UK). (b) Examples of clot density scores from a mastitis detection field study in New Zealand (from Kamphuis et al., 2013a). Color version available online. 
for $\mathrm{CM}$ during a time window running from 2 milkings before the first clots of a CM episode appear until the $\mathrm{CM}$ episode ends. The $\mathrm{Se}_{\text {overall }}$, thus, represents how accurate a sensor is in finding cows with CM (Table 2). The calculation was repeated using a narrower time window to calculate timely sensitivity $\left(\mathbf{S e}_{\text {timely }}\right)$. For $\mathrm{Se}_{\text {timely }}$, a sensor is expected to alert at least once for CM during a time window running from 2 milkings before to 1 milking after the first clots of a $\mathrm{CM}$ episode appeared (4 milkings in total). The $\mathrm{Se}_{\text {timely }}$, thus, provides an indication of how accurate and timely a sensor is in detecting CM (Table 2). Both sensitivities were computed per sensor system per farm, but also per sensor system across farms. Sensor performance for detecting $\mathrm{CM}$ in the current study was evaluated as per the protocol: thresholds were used on the sensor data to generate CM alerts. These alert thresholds were set such that they resulted in approximately 10 false alerts per 1,000 cow milkings (FAR1000; Table 2). Figure 2 graphically explains how alerts from a hypothetical mastitis-detection system should be labeled, and how this labeling should be used to calculate sensitivity and FAR1000. This example is extracted from several examples provided by Kamphuis et al. (2013a).

\section{Requirement 2: Identifying Cows with High SCC to Manage BMSCC}

Data were collected on the same farms used for requirement 1 . The milk recording test day was used as the gold standard, and, for the current study, test day recording comprised 4 consecutive cow milkings starting at the evening milking. Test day recording was conducted by a certified milk recording provider (Livestock Improvement Corporation, Hamilton, New Zealand) according to New Zealand Standard (2007).

Table 2. Description and abbreviations of terms used to evaluate in-line mastitis detection systems for the first requirement (detecting clinical mastitis promptly and accurately; adapted from Kamphuis et al., 2013a)

\begin{tabular}{|c|c|c|}
\hline Term & Abbreviation & Description \\
\hline Time window $^{1}$ & - & $\begin{array}{l}\text { The time frame in which a mastitis-detection system should generate an alert. This time } \\
\text { period should be } 4 \text { cow milkings and is centered on the start of the milking with the first } \\
\text { clot observation (i.e., a time-window of } 2 \text { cow milkings before the first cow milking with } \\
\text { clots in a confirmed CM episode and } 1 \text { cow milking after the first cow milking with clots } \\
\text { in a confirmed CM episode). }\end{array}$ \\
\hline True positive alert $^{2}$ & $\mathrm{TP}$ & $\begin{array}{l}\text { A first alert generated by a mastitis-detection system that occurs within a time-window } \\
\text { of } 4 \text { cow-milkings around the start of a confirmed CM episode. }\end{array}$ \\
\hline Late true positive alert ${ }^{3}$ & Late TP & $\begin{array}{l}\text { A late TP alert occurs when the mastitis-detection system alerts for the first time within } \\
\text { a CM episode but outside the time-window of } 4 \text { cow milkings around the start of a } \\
\text { confirmed CM episode. }\end{array}$ \\
\hline Confirmative alerts & - & $\begin{array}{l}\text { Alerts that occur within } 2 \text { cow milkings after a TP or late TP or that fall within time } \\
\text { window of } 2 \text { cow-milkings of another confirmative alert. }\end{array}$ \\
\hline Sensitivity & Se & $\begin{array}{l}\text { The Se refers to the proportion of CM episodes that are correctly detected by a mastitis- } \\
\text { detection system. }\end{array}$ \\
\hline Timely sensitivity & $\mathrm{Se}_{\text {timely }}$ & $\begin{array}{l}\text { The } \mathrm{Se}_{\text {timely }} \text { refers to the proportion of } \mathrm{CM} \text { episodes that are correctly and promptly } \\
\text { detected by a mastitis-detection system within the required } 48 \text {-h time window. Calculated } \\
\text { as: } \mathrm{Se}=[\mathrm{TP} /(\mathrm{TP}+\mathrm{FN})] \times 100 \text {. }\end{array}$ \\
\hline Overall sensitivity & $\mathrm{Se}_{\text {overall }}$ & $\begin{array}{l}\text { The Se } \mathrm{Sverall}_{\text {refers to the proportion of } \mathrm{CM} \text { episodes that are correctly detected by a }} \\
\text { mastitis-detection system. Calculated as: } \mathrm{Se}=[(\mathrm{TP}+\text { Late } \mathrm{TP}) /(\mathrm{TP}+\text { Late } \mathrm{TP}+\mathrm{FN})] \\
\times 100 \text {. }\end{array}$ \\
\hline $\begin{array}{l}\text { False alerts per } 1,000 \\
\text { cow milkings }\end{array}$ & FAR1000 & $\begin{array}{l}\text { The FAR1000 refers to the number of FP alerts per } 1000 \text { cow-milkings. Calculated as: } \\
\text { FAR1000 }=(1,000 \times \mathrm{FP}) / \text { total cow milkings. }\end{array}$ \\
\hline
\end{tabular}

${ }^{1}$ Based on the workshop, this time window is extended to 5 milkings; that is, a time window of 2 milkings before the milking with the first clot observation of a CM episode, the milking with the first clot observation of a CM episode, and 2 milkings after the milking with the first clot observation of a CM episode.

${ }^{2}$ Based on the workshop, a true positive alert occurs within a time window of 5 cow milkings around the start of a confirmed CM episode.

${ }^{3}$ Based on the workshop, the definition of a late true positive alert is changed by having the an alert for the first time within a CM episode, but outside the time window of 5 cow milkings and limited to a maximum of $10 \mathrm{cow}$ milkings starting at the milking where clots were observed for the first time.

${ }^{4}$ Based on the workshop, a false positive alert is any alert generated by a mastitis-detection system that falls outside the time window of 5 cow milkings and outside the remainder of the CM episode and that is not a confirmative alert. 


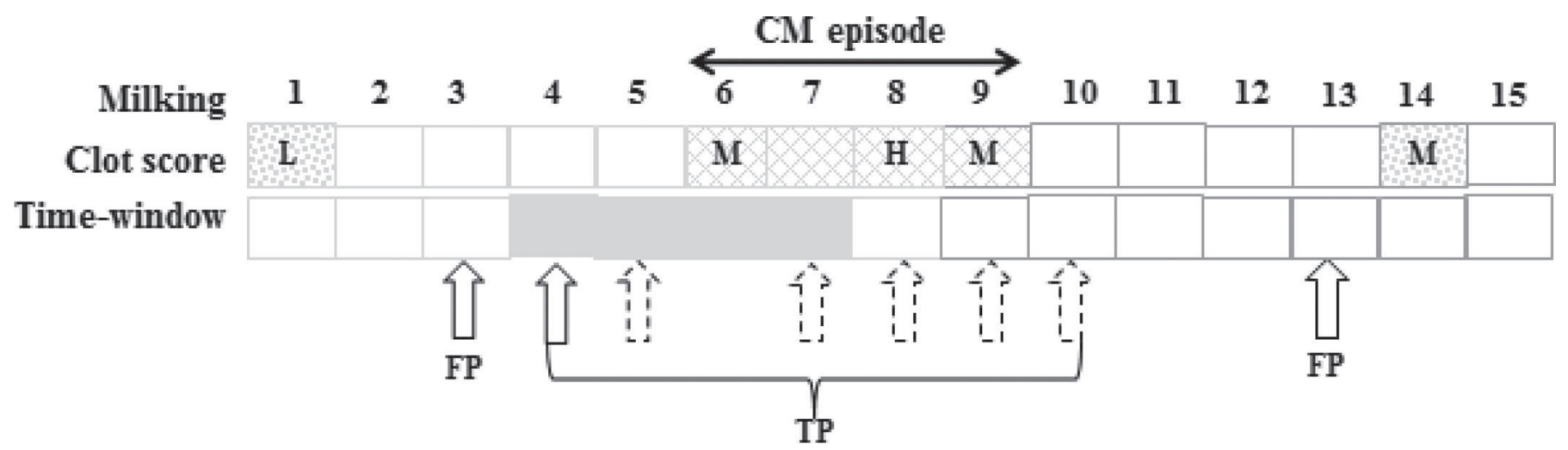

Figure 2. Applying the proposed gold standard to identify clinical mastitis (CM) episodes and using time window analyses to link CM episodes with alerts generated by a hypothetical mastitis-detection system. The example consists of 15 consecutive cow milkings in which 5 have clots that were scored for density (L, M, or H representing low, medium, and high, respectively). One CM mastitis episode was observed (milking 6 through 9) and the time window of 4 cow milkings in which an alert was expected is indicated by dark gray blocks (cow milking 4 through 7 ). The mastitis-detection system generated several alerts. Two of them clearly fell outside the CM episode (cow milking 3 and 13 ) and are, thus, considered false positives (FP). The first alert falling within the required time window is generated at cow milking 4 , and this true positive alert (TP) is followed by a series of confirmative alerts (dashed arrows) within the time window of 4 cow milkings. These are combined into 1 TP alert; the alerts on milking 8,9, and 10 are confirmative alerts, as they occurred within 2 cow milkings of an alert that was merged with a TP alert. The TP alerts, FP alerts, and the number of cow milkings are used to calculate sensitivity (Se) and the false alert rate per 1,000 cow milkings (FAR1000). In doing so, milking 1 and 14 are ignored, as these involve single clot observations. The alert at milking 13 is also ignored, as this alert is generated 1 cow milking before the single clot observation at milking 14 . All in all, this leaves $1 \mathrm{FP}$ at cow milking 3,1 timely TP alert at cow milking 4, and 13 cow milkings to be included for FAR1000. The hypothetical mastitis-detection system, thus, has a timely Se of $100 \%$ and a FAR1000 of 77. From Kamphuis et al. (2013a).

Results from milk recording test days were retrieved from the provider and included cow identification number, milk yield, milk composition (fat, protein, lactose, and SCC), date of milk recording, and session of milk recording (morning or evening session).

Milking data collected from the herd-management software were the same as in requirement 1: cow identification number, stall number, date and time, milking session, and sensor measurements. Sensor measurements were again not corrected for any outliers.

Milk recording test day data and herd management milking data were merged by date, milking session, and cow identification number. Data on milk volume and SCC from milk recording test days were used as the gold standard. For each cow, the total SCC contribution to the bulk milk was computed by multiplying reported milk volume with SCC content. This total SCC contribution was used to rank cows, where cows with the highest SCC contribution were ranked 1. Using the same data, BMSCC was estimated by summing all individual cow SCC contributions and dividing this by the total volume of milk produced. Cows were also ranked according to the outcome of the sensors (e.g., highest EC or SCC values were ranked 1). Both rankings were used to create ranking curves (Kamphuis et al., 2013a) by plotting relative reduction in BMSCC by excluding each cow one by one, starting with rank 1. The percentage of cows to be excluded that reduced BMSCC by $25 \%$ was determined for both systems. Analysis for requirement 2 were conducted by combin- ing all data from all cow milkings (analyzing them as 1 milk recording test day), but also per farm (analyzing 4 milking sessions per farm as 1 milk recording test day) and per farm per milking session.

\section{Review of the Protocols by Technology Suppliers}

Results from the field validation were summarized and presented at a workshop in April 2013 (Hamilton, New Zealand) with representatives from 7 main companies supplying milking and animal-monitoring technologies in New Zealand. Companies attending included GEA Farm Technologies (Hamilton, New Zealand), Waikato Milking Systems (Hamilton, New Zealand), Livestock Improvement Corporation (Hamilton, New Zealand), Gallagher Group (Hamilton, New Zealand), Dairy Automation Limited (Hamilton, New Zealand), Lely Sensortec (Hamilton, New Zealand), and TruTest (Auckland, New Zealand). Companies that were invited but were unable to attend the workshop included DeLaval, DairyMaster, and Lely Industries. In addition, 3 farmers and 3 representatives of the dairy industry were present, as well as a precision dairy researcher from Massey University (Palmerston North, New Zealand). The workshop was a critical element of our study and was aimed at gathering industry critique and support of the protocols at a New Zealand dairy industry level. Participants were sent a copy of the protocols before the workshop, and were invited to comment on them. During the workshop itself, each section of the proto- 
Table 3. Number of cows with 1 or more clinical mastitis (CM) episodes, number of CM episodes, and number (percentage) of clot observations within and outside a CM episode ${ }^{1}$

\begin{tabular}{lcccc}
\hline Farm & $\begin{array}{c}\text { Cows with } \\
1 \text { or more } \\
\text { CM episode }\end{array}$ & $\begin{array}{c}\text { CM } \\
\text { episodes }\end{array}$ & $\begin{array}{c}\text { Clots within CM } \\
\text { episodes (\% of total } \\
\text { clot observations }\end{array}$ & $\begin{array}{c}\text { Clots outside CM } \\
\text { episodes (\% of total } \\
\text { clot observation }\end{array}$ \\
\hline 1 & 31 & 35 & $97(39)$ & $149(61)$ \\
2 & 42 & 56 & $177(38)$ & $284(62)$ \\
3 & 15 & 19 & $51(35)$ & $96(65)$ \\
Total & 88 & 110 & $325(38)$ & $529(62)$ \\
\hline
\end{tabular}

${ }^{1} \mathrm{~A}$ CM episode is defined as the observation of clots at 2 out of 3 , or at all 3 , consecutive milkings (see Table 2).

${ }^{2}$ The total number of clot observations are listed in Table 1.

cols was explained and illustrated with the results from the field study. During each section, the protocols were discussed and feedback and consensus were achieved. Notes were taken to record comments provided during the workshop. After the workshop ended, participants who had not commented on the protocols were requested to do so via mail within a month. Comments and notes were used in the evaluation of the protocols.

In preparation for the workshop, additional analyses were conducted for both requirements. For requirement $1, \mathrm{Se}_{\text {overall }}$ and $\mathrm{Se}_{\text {timely }}$ were calculated by combining all collected data as per the protocol, but these were also calculated per farm. Also, $\mathrm{Se}_{\text {overall }}$ and $\mathrm{Se}_{\text {timely }}$ were both calculated at additional levels of FAR1000 (at 20, 50, and 100 false alerts per 1,000 cow-milkings). Analyses were also conducted excluding the low-density clot observations, as in practice farmers may not consider lowdensity clots to represent genuine clinical infections. The exclusion of low-density clots was only conducted using data from all 3 farms combined, not for each farm separately.

The protocol for requirement 2 does not set any requirements on BMSCC levels of farms. The 3 farms with in-line SCC sensors enrolled for the field validation of requirement 1 all had a low $\operatorname{BMSCC}(<150 \times$ $10^{3}$ cells $/ \mathrm{mL}$ ). To assess the robustness of results for requirement 2 , data collection and analysis were also conducted on 3 additional farms with a relatively high BMSCC $(>250,000$ cells $/ \mathrm{mL})$. These 3 farms were selected from farms that participated in a milking efficiency study (Edwards et al., 2013) and were known to have rotary milking systems, herd sizes $>500$ cows, and a BMSCC at the most recent milk recording test day $>250,000$. These farms had sensor systems recording the average $\mathrm{EC}\left(\mathbf{E C}_{\text {mean }}\right)$ at every stall, but inline SCC sensors were not installed. Details on all 6 farms that were included to validate requirement 2 are reported in Table 6. All analysis (those proposed by the protocol and those added in preparation for the workshop) were conducted on all 6 farms; SAS (version 9.3, SAS Institute Inc., Cary, NC) was used for all data preparation and analysis.

\section{RESULTS}

\section{Requirement 1: Identifying CM Promptly and Accurately}

A total of $110 \mathrm{CM}$ episodes (range $=19-56$ per farm) were recorded from 88 cows (range $=15-42$ per farm; Table 3). Thirty-eight percent of all clot observations were within a $\mathrm{CM}$ episode. These percentages were consistent across farms (Table 3). The number of CM episodes per cow ranged from 1 to 4 on farms 1 and 2 , and from 1 to 3 on farm 3 .

Density scores of the 854 clot observations that could be merged with milking data are summarized in Table 4. The proportion of high-density clots was relatively high on farm 1 compared with farms 2 and 3. Of the 529 clot observations falling outside a CM episode (Table 3), 258 (49\%), 208 (38\%), and 63 (12\%) had a low-, medium-, or high-density score, respectively. Of the 325 clot observations falling inside a CM episode (Table 3), 85 (26\%), 119 (37\%), and 121 (37\%) had a low-, medium-, or high-density score, respectively.

Overall and timely Se values for several levels of FAR1000 for both the $\mathrm{EC}_{\max }$ sensor and the in-line SCC sensor are summarized in Table 5. The expected trend of increased Se values with increasing FAR1000 was confirmed for both sensors, as well as the expected trend that $\mathrm{Se}_{\text {overall }}$ would outperform $\mathrm{Se}_{\text {timely }}$. Also, large variation was observed in Se levels across farms (e.g., at a FAR1000 of $10 \mathrm{Se}_{\text {timely }}$ varied between 20 and 43 for $\mathrm{EC}_{\max }$, and between 5 and 43 for in-line SCC). Neither sensor system met the proposed minimum target of $80 \%$ Se at a FAR1000 of 10 (Kamphuis et al., 2013a) or at higher levels of FAR1000. Farm 3 differed from the other 2 farms, as little difference was measured between $\mathrm{Se}_{\text {overall }}$ and $\mathrm{Se}_{\text {timely }}$ at the same FAR1000 and Se levels for in-line SCC were much lower. Similar trends were observed when FAR1000 was fixed at 10 and all data were combined and analyzed as 1 farm. Sensitivity levels $>50 \%$ were only met by $\mathrm{EC}_{\max }$ and only at higher levels of FAR1000. Ignoring clot observations with a low-density score reduced the number of cow milkings 
Table 4. Number (percentage) of clot observations that received a low-, medium-, or high-density score, and the total number of clot observations, per farm

\begin{tabular}{lrrrr}
\hline & \multicolumn{3}{c}{$\begin{array}{c}\text { Number (\%) of clot observations } \\
\text { with density score }\end{array}$} & $\begin{array}{c}\text { Total clot } \\
\text { observations }\end{array}$ \\
\cline { 2 - 4 } Farm & \multicolumn{1}{c}{ Low } & Medium & High & 246 \\
1 & $46(19)$ & $97(39)$ & $103(42)$ & 461 \\
2 & $210(45)$ & $183(40)$ & $68(15)$ & 147 \\
3 & $87(59)$ & $47(32)$ & $13(9)$ & 854 \\
Total & $343(40)$ & $327(38)$ & $184(22)$ & \\
\hline
\end{tabular}

included in the analysis slightly and reduced the number of CM episodes to 68 . Trends were the same as in those analyses including all clot observations, but Se levels were around 10 percentage points higher when low-density clots were excluded. The $\mathrm{Se}_{\text {overall }}$ was close (78\%) to the target of $80 \%$ for $\mathrm{EC}_{\max }$ when FAR1000 was increased to 100 .

\section{Requirement 2: Identifying Cows with High SCC to Manage BMSCC Levels}

Table 6 summarizes details of all 6 farms included for field validation of the second requirement of automated in-line mastitis-detection systems. Although the farms chosen for a high BMSCC did not exceed the selection criterion of $>250,000$ cells $/ \mathrm{mL}$ for all milk recording test days, they had a higher BMSCC than the other 3 farms (Table 6).

Over $90 \%$ of cow milkings with milk recording test day SCC also had an EC value recorded. As in-line SCC sensors were installed on every fourth stall, only one quarter of all cow-milkings with test-day SCC information also had an in-line SCC estimate (Table 6). Of the 4,344 cows included in the analysis (Table 6), only 4 cows $(<0.1 \%)$ did not have a single cow milking with an EC measurement. Of the 2,578 cows from

Table 5. Performance of sensors measuring the maximum electrical conductivity $\left(\mathrm{EC}_{\max }\right)$ at whole-udder level at every stall or measuring inline SCC at every fourth stall in identifying cows with clinical mastitis (CM) episodes (requirement 1) at 4 levels of false alerts per 1,000 cow milkings $(\text { FAR } 1000)^{1}$

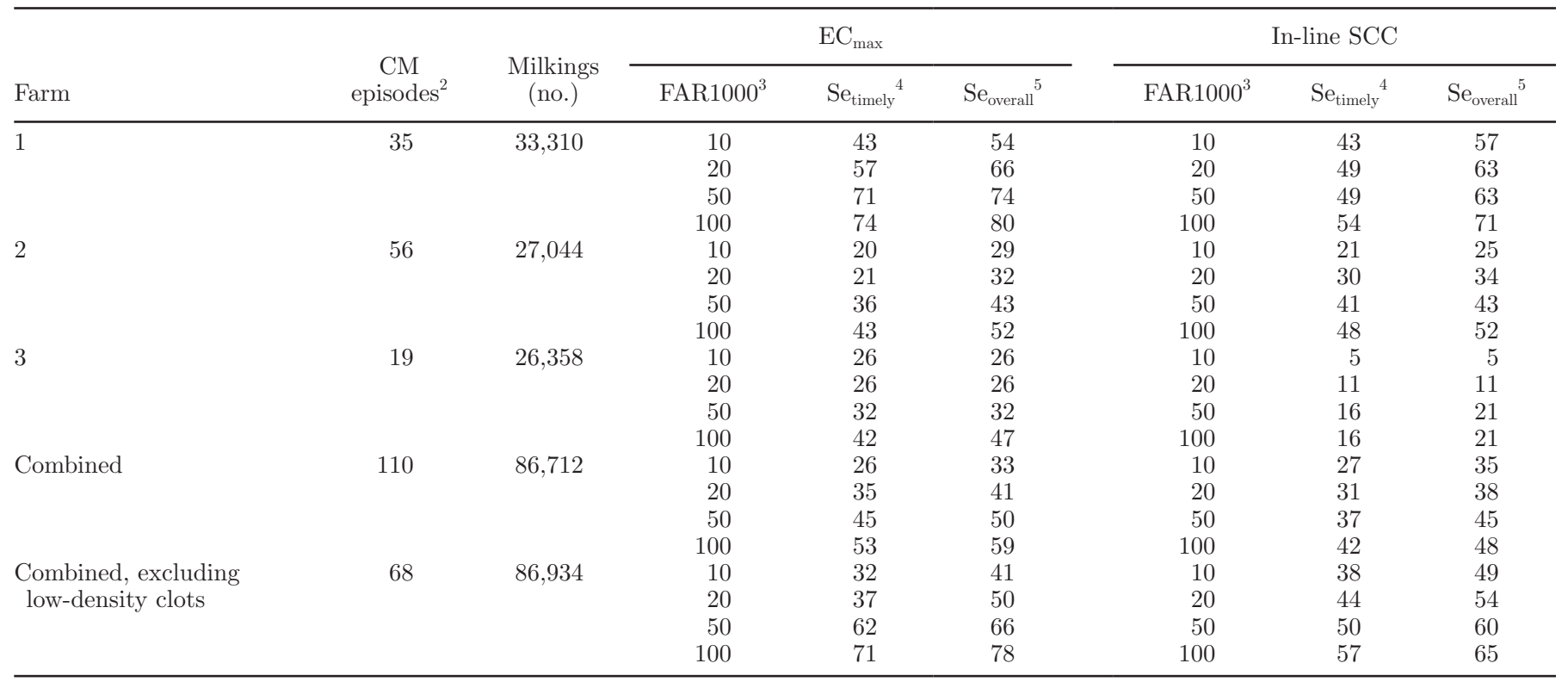

${ }^{1}$ Two sensitivity levels are reported for each FAR1000: timely sensitivity $\left(\mathrm{Se}_{\text {timely }}\right)$ and overall sensitivity $\left(\mathrm{Se}_{\text {overall }}\right)$. Results are reported per farm and for all 3 farms combined. The combined analysis was conducted using all clot observations, and when low-density clots were excluded.

${ }^{2} \mathrm{~A}$ clinical mastitis (CM) episode is defined as the observation of clots at 2 out of 3, or at all 3, consecutive milkings (see Table 2).

${ }^{3}$ The false alerts per 1,000 cow milkings (FAR1000) refers to the number of false positive alerts per 1,000 cow milkings (see Table 2).

${ }^{4}$ The timely sensitivity $\left(\mathrm{Se}_{\text {timely }}\right)$ refers to the proportion of $\mathrm{CM}$ episodes that are correctly and promptly detected by a mastitis-detection system within the required time window of 4 cow milkings (see Table 2).

${ }^{5}$ The overall sensitivity $\left(\mathrm{Se}_{\text {overall }}\right)$ refers to the proportion of $\mathrm{CM}$ episodes that are collected detected by a mastitis-detection system (see Table 2 ). 


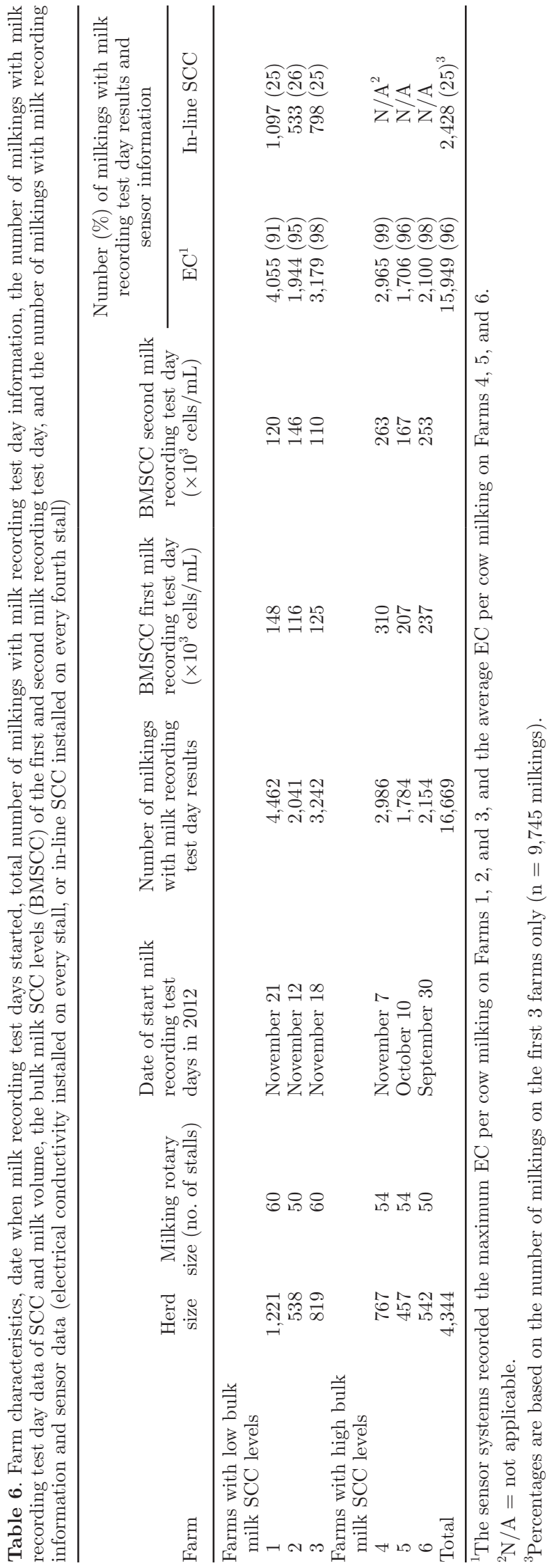

farms with in-line SCC sensors installed (Table 6), 833 cows $(32 \%)$ did not have a single cow milking with an in-line SCC measurement. These cows (and their milkings) remained in the data set, but, when ranked, cows without sensor information simply ranked at the bottom of the ranking list.

The overall BMSCC for the 3 low-SCC farms averaged $128 \times 10^{3}$ cells $/ \mathrm{mL}$. To reduce this BMSCC by $25 \%, 0.7 \%$ of the cows, those highest ranked, had to be excluded (Table 7). When based on $\mathrm{EC}_{\max }$ or on in-line SCC measurements, these percentages increased to 6.6 and $25.4 \%$, respectively (Table 7 ), which was more than twice the percentage indicated by the gold standard. Therefore, on these farms, neither sensor system met the minimum performance requirements proposed by Kamphuis et al. (2013a). Similar exclusion percentages were required when the ranking was made on pooled data of farms 1 and 2 or per milking session for farms 1 and 2. In contrast, farm 3 consistently returned higher exclusion percentages when ranking was based on $\mathrm{EC}_{\max }(13$ vs. $6 \%$ on farm 1 and 2; Table 7).

Generally, $\sim 25 \%$ of the highest-ranking cows required exclusion in herds when the ranking was based on inline SCC measurements, the exception being milking 4 for farm 2. When including data only from stalls with a SCC sensor, ranking due to the in-line SCC system improved markedly. In this scenario, the BMSCC was recalculated as $134 \times 10^{3}$ cells $/ \mathrm{mL}$ using the data from 2,248 cow milkings. The gold standard ranking method indicated that $0.9 \%$ of the herd required exclusion to reduce BMSCC by $25 \%$. This compared with 4 and $2 \%$ of the herd when ranking was based on $\mathrm{EC}_{\max }$ or in-line SCC values, respectively (Table 7; Figure 3). In this case, in-line SCC met the proposed requirement of not excluding more than twice the percentage indicated by the gold standard method.

For the 3 farms with a high BMSCC (Table 8), the highest ranked $1 \%$ of the herd had to be excluded when ranking was based on the gold standard method. Ranking based on $\mathrm{EC}_{\text {mean }}$ consistently required exclusion of more than twice the percentage indicated by the gold standard method. This averaged $15 \%$ when pooling all the data, which was higher than for the low-BMSCC farms. However, pooling the data per farm demonstrated a large variation between farms: from $9 \%$ for farm 6 to $96 \%$ for farm 5 . In the latter case, 2 cows were major contributors to the BMSCC, being ranked 2 and 11 based on the gold standard method, but they had no $\mathrm{EC}_{\text {mean }}$ measured. As a consequence, these cows were ranked at the bottom of the ranking list and a large proportion of the herd had to be excluded to achieve the required $25 \%$ reduction in BMSCC. 


\section{DISCUSSION}

The current study used 2 different sensors to validate protocols for assessing the performance of automated inline mastitis-detection systems. It is important to note that the results reported are based on the application of simple thresholds on sensor data to generate mastitis alerts (for requirement 1) and unmodified sensor data to rank cows (for requirement 2). The equipment suppliers were asked to provide unmodified sensor data, as the study objective was to evaluate the practicality of the protocols, not to evaluate the field performance of sensors. In reality, the suppliers have proprietary algorithms that process sensor data and, perhaps, combine data from several sensors to generate alerts. Thus, the results reported are likely to be an underestimation of performance. When the protocols are to be used to evaluate performance of a mastitis-detection sensor (or sensor system), the only information required from the supplier is a sensor-derived mastitis alert list, and a ranking of the cows. Another important note is that the protocols are all about assessing the performance of automated in-line mastitis-detection systems. Actions that should be taken by farmers in response to the in- formation that is provided by these detection systems are not considered in these protocols and, thus, not in this study to validate the protocols.

After applying the proposed protocol for requirement 1, $110 \mathrm{CM}$ episodes were identified during $\sim 3 \mathrm{wk}$ of observation per farm, roughly equivalent to $9 \mathrm{CM}$ episodes per 100 cows per month. The high level of CM incidence was strongly influenced by farm 2 , which had a CM incidence of 15 episodes per 100 cows per month based on the clots observed. The incidence of $\mathrm{CM}$ on farms 1 (6 CM episodes per 100 cows per month) and 3 (3 CM episodes per 100 cows per month) were more in line with previously reported results.

Previous mastitis-detection research has often reported performance using Se and Sp. The Sp and FAR1000 are related (Sherlock et al., 2008), but FAR1000 should be easier for farmers to interpret. The reported FAR1000 levels of $10,20,50$, or 100 in the current study approximate to Sp levels of 99, 98, 95, and 90\%, respectively. As sensitivity increased, the FAR1000 levels increased and specificity decreased. This trend has been reported previously in studies of the detection of events such as mastitis (Miekley et al., 2013), estrus (Hockey et al., 2010), and lameness (Kamphuis

Table 7. For farms with lower bulk milk SCC (BMSCC) levels (farm 1, 2, and 3), the number of milkings included to calculate BMSCC, the lower 5\% and upper $95 \%$ limits for milk recording SCC, and the percent of the herd that had to be excluded to reduce the BMSCC by $25 \%$ when ranking of cows was done based on their BMSCC contribution (gold standard), or on sensor output (electrical conductivity ( $\mathrm{EC}_{\text {max }}$ ) or in-line SCC $]^{1}$

\begin{tabular}{|c|c|c|c|c|c|c|}
\hline \multirow[b]{2}{*}{ Analysis } & \multirow[b]{2}{*}{$\begin{array}{l}\text { Milkings } \\
\text { (no.) }\end{array}$} & \multirow[b]{2}{*}{$\begin{array}{c}\text { BMSCC } \\
\left(\times 10^{3} \text { cells } / \mathrm{mL}\right)\end{array}$} & \multirow{2}{*}{$\begin{array}{l}\text { Upper and lower } \\
\text { limit of SCC } \\
\left(\times 10^{3} \text { cells } / \mathrm{mL}\right)\end{array}$} & \multicolumn{3}{|c|}{$\begin{array}{l}\text { Percent of the herd excluded } \\
\text { when ranking is based on }\end{array}$} \\
\hline & & & & $\begin{array}{l}\text { BMSCC } \\
\text { contribution }\end{array}$ & $\mathrm{EC}_{\max }$ & $\begin{array}{l}\text { In-line } \\
\text { SCC }\end{array}$ \\
\hline Combined & 9,745 & 128 & $125-149$ & 0.7 & 6.6 & 25.4 \\
\hline \multicolumn{7}{|l|}{ By farm } \\
\hline Farm 1 & 4,462 & 135 & $122-157$ & 0.6 & 6.1 & 25.0 \\
\hline \multicolumn{7}{|l|}{ By farm and milking session (S) } \\
\hline \multicolumn{7}{|l|}{ Farm 1} \\
\hline S1 & 1,057 & 160 & $121-187$ & 0.8 & 5.8 & 24.2 \\
\hline $\mathrm{S} 2$ & 1,181 & 142 & $108-178$ & 0.5 & 4.7 & 25.1 \\
\hline S3 & 1,062 & 141 & $106-181$ & 0.8 & 8.8 & 23.2 \\
\hline $\mathrm{S} 4$ & 1,162 & 108 & $85-152$ & 0.6 & 4.7 & 26.1 \\
\hline \multicolumn{7}{|l|}{ Farm 2} \\
\hline \multicolumn{7}{|l|}{ Farm 3} \\
\hline $\mathrm{S} 1$ & 812 & 162 & $122-191$ & 1.0 & 4.2 & 25.4 \\
\hline $\mathrm{S} 2$ & 811 & 98 & $76-177$ & 1.0 & 18.9 & 24.8 \\
\hline S3 & 808 & 132 & $104-155$ & 1.2 & 11.3 & 25.5 \\
\hline S4 & 811 & 95 & $71-125$ & 0.9 & 21.0 & 25.5 \\
\hline $\begin{array}{l}\text { Combined including only milkings with } \\
\text { values for both sensor systems }\end{array}$ & 2,428 & 134 & $119-173$ & 0.9 & 3.9 & 1.7 \\
\hline
\end{tabular}


Table 8. For farms with higher bulk milk SCC (BMSCC) levels (farm 4, 5, and 6), the number of milkings included to calculate BMSCC, the lower 5\% and upper 95\% limits for milk recording SCC, and the percent of the herd that had to be excluded to reduce this BMSCC by $25 \%$ when ranking of cows was done based on their bulk milk SCC contribution (gold standard) or on sensor output mean electrical conductivity $\left(\mathrm{EC}_{\text {mean }}\right)^{1}$

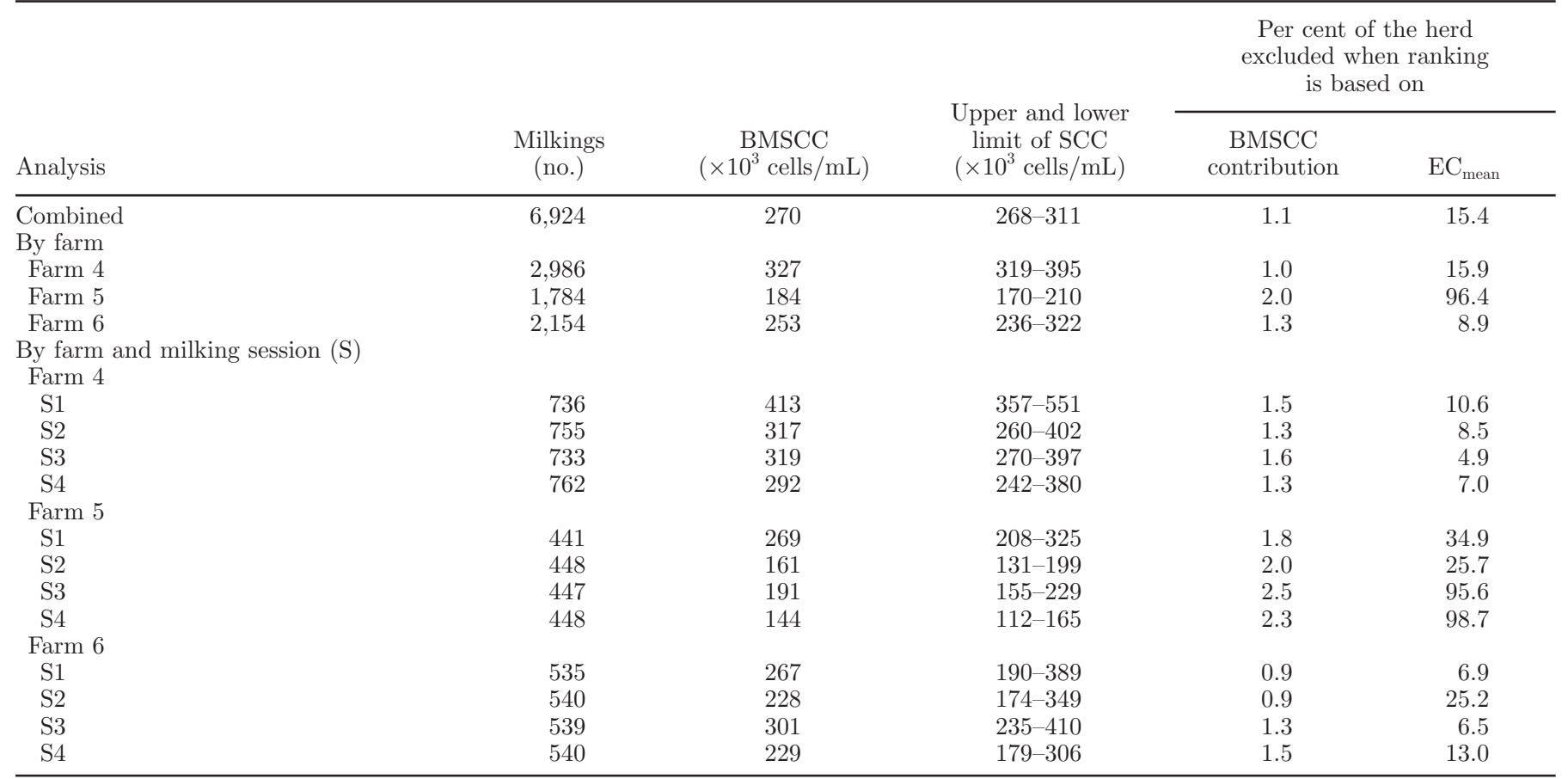

${ }^{1}$ Results are presented when data from all 3 farms were pooled and analyzed as one milk recording test day (combined), by farm, and by farm and milking session.

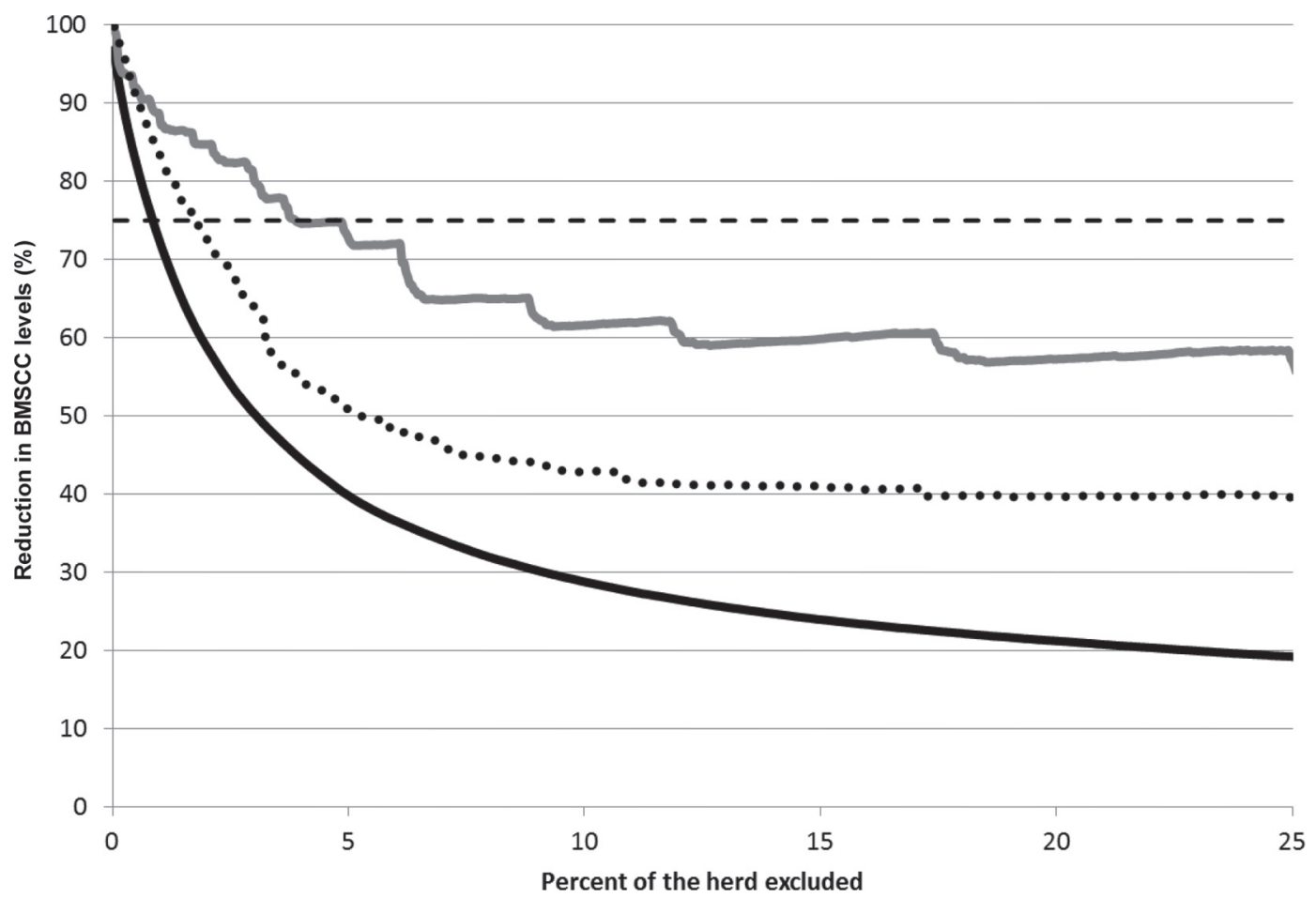

Figure 3. Ranking curves for the gold standard (solid black line; ranking based on SCC contribution to the bulk milk), for electrical conductivity max (solid gray line), and for in-line SCC (dotted black line) using only records with milk recording test-day information and with in-line SCC measurements. The dashed horizontal black line represents the $25 \%$ reduction in bulk milk SCC (BMSCC) levels. 
et al., 2013b). The practicality of increasing levels of FAR1000 (or decreasing levels of specificity) is an operational decision for individual farmers. A FAR1000 of 10 means that a farmer has to manage 10 false alerts per day for a 500-cow herd, or 2 false alerts per day for a 100-cow herd (if milked twice daily). Farmers may accept a higher false alert rate, but it is likely that a FAR1000 of 100 will be too high for many farmers, particularly those with automatic milking systems, as they aim to minimize false alerts to avoid fetching cows unnecessarily (Mollenhorst et al., 2012).

The 2 sensors in this field validation study both demonstrated a wide range in performance between farms. Performance of the sensors in detecting CM episodes was much better on farm $1\left(54 \% \mathrm{Se}_{\text {overall }}\right.$ at FAR1000 of 10 for $\mathrm{EC}_{\max } ; 57 \% \mathrm{Se}_{\text {overall }}$ at FAR1000 of 10 for in-line SCC) compared with the other 2 farms. A variation in automated in-line mastitis-detection performance between farms has been reported before (Kamphuis et al., 2010b). Differences in detection performance were explained by Kamphuis et al. (2010b) as farmers either being structured and organized in using information from mastitis alert lists compared with farmers who were more chaotic and less structured in the identification of cows showing clots in milk. This does not explain the variation found in the current study, as all cow milkings were assessed for the presence of clots. However, differences between farms may be explained by variance in the CM episode severity. Assuming that clot density scores provide information on the severity of a CM infection, where higher scores indicate a more severe CM, then the CM episodes on farm 1 (with more high-density clots observed) were more severe than those on farms 2 and 3 (with relatively few high-density clot observations). More severe cases of $\mathrm{CM}$ are likely to increase EC and SCC more significantly compared with mild cases of $\mathrm{CM}$, making detection of these $\mathrm{CM}$ easier. This explanation was supported by excluding low-density clots, resulting in improved Se values for both sensors compared with the analysis where lowdensity clots were included.

There was a large variation in the proportion of cows excluded from the bulk milk to reduce BMSCC by $25 \%$ when using EC measurements, and this variation was more extreme on farms with a higher BMSCC. One possible reason for this is the failure to calibrate the sensors regularly. Calibration is an important prerequisite for good sensor performance, but is often neglected. Recent research demonstrated that one third of 83 users of milk meters had not calibrated them since installation (Eastwood et al., 2015). For the in-line SCC sensor, the high proportion of cows that had to be excluded to reduce BMSCC by $25 \%$ was largely due to the fact that the sensors were only installed on every fourth stall instead on every stall. Installation of sensors at every second or fourth stall was considered by the supplier to be an acceptable compromise between investment costs and system performance, but in our study this resulted in $75 \%$ of cows not having an SCC measurement at each milk recording test day milking. These cows were not excluded from the analysis, but instead were ranked below those that did receive an SCC estimate, which negatively affected the result for the second requirement. To demonstrate the potential of the in-line SCC system when installed on every stall, only records with in-line SCC measurements were used in the analysis. This resulted in the percentage of the herd to be excluded to reduce the BMSCC by $25 \%$ dropping from 25 to $2 \%$ of the highest-ranked cows. The choice to reduce capital costs by installing fewer in-line SCC sensors has to be weighed against the extended time required to identify high-SCC cows for managing BMSCC levels. Moreover, the installation also depends on how the inline SCC is used: installing fewer in-line SCC sensors may still be appropriate in those situations where the farmer uses the information to support management decisions (e.g., culling of cows with chronically high SCC levels). However, in situations where these in-line SCC sensors are used for automatically diverting poorquality milk (automatic dumping), a sensor would be required on each stall.

This study was conducted to evaluate practicality of the protocols. During the field validation of both protocols, no unexpected issues were identified nor did impractical situations occur. The protocol for requirement 2 was easily implemented: data collection for the gold standard can be done by the milk recording test day provider and no further expertise is required. The protocol for requirement 1 was more labor intensive (with associated costs) over an extended period (3-4 wk), so this protocol did require more organizational skills. Instructing the on-farm personnel to record all observed clots reduced interpretation errors and training requirements. Experience from the current field validation demonstrated that hiring and training personnel worked better than using existing farm staff for collecting clot observations. Farm staff (on farm 1) were prone to inattention when monitoring the in-line filter if the milking process got interrupted for any reason, whereas the trained external personnel (farm 2 and 3 ) stayed focused on their assigned task.

The data collection highlighted some practical considerations, specifically the process for dealing with missing or incorrect records. Common examples included records without related test-day results for SCC or milk volume, cow milkings without sensor information, mismatches between cow and stall number recorded on photos of clot observations, or with data 
extracted from the herd management software. When evaluating requirement 1 , clot observations that could not be matched by cow identification number, stall number, date, and milking session were excluded from further analysis, which resulted in a loss of $11 \%(13$, 15 , and $5 \%$ for farms 1,2 , and 3, respectively) of all clot observations. When this protocol is implemented in future, careful attention should be paid to recording cow identification and stall numbers to minimize loss of data. On farm 3, the largest proportion of clot observations were lost (45\%) due to software issues, as in many instances milking data were not recorded. In validation of requirement 2 , data losses were due mainly to the absence of in-line SCC on each stall.

There has been uncertainty as to whether technology suppliers would accept the costs and labor associated with the requirement 1 (Kamphuis et al., 2013a). However, technology supplier representatives expressed general support for the protocols in providing robust and consistent information that farmers would understand. Technology suppliers involved in the workshop also suggested 4 structural refinements of the protocol. First, they were in favor of using only medium- and high-density clots, as it was considered that this would link more closely with farmer practice (low-density clots may not be recognized as signs of $\mathrm{CM}$ requiring attention by farmers or farm staff). Therefore, we recommend adjusting the protocols to include the option to record clot density. Where clot density is scored, the performance analysis should include all clot observations, along with an additional analysis that excludes low-density clots (as in the current study). Where density is not recorded, analysis should be done using all clot observations, regardless of their density. The second recommendation was to extend the time window of 4 cow milkings in which an alert was expected for $\mathrm{Se}_{\text {timely }}$ to 5 cow milkings. This would include alerts that occur 2 milkings before the milking with the first clot observation of a CM episode, the milking with the first clot observation of a CM episode, and 2 milkings after the milking with the first clot observation of a CM episode. Extending the time window to 5 cow milkings for $\mathrm{Se}_{\text {timely }}$ influenced results positively: all Se values improved with a maximum of 6 percentage points for EC and with a maximum of 3 percentage points for in-line SCC (results not shown) when data across farms were combined. The third change was to set a limit on the number of cow milkings in the time window in which a mastitis-detection system is expected to alert for CM for $\mathrm{Se}_{\text {overall }}$. The protocol labels an alert as a late true positive result where a mastitis-detection system alerts for the first time within a CM episode but outside the time window for $\mathrm{Se}_{\text {timely }}$ (Kamphuis et al., 2013a). In theory, CM episodes could last for a long period and timeliness in mastitis detection is important. Limiting the definition of a late true positive alert to a maximum of 10 cow milkings starting at the milking where clots were first observed appears reasonable. The fourth suggested change to the protocol was based on the proposed performance standard reported in Kamphuis et al. (2013a). The technology suppliers suggested excluding the minimum performance criterion (a minimum $\mathrm{Se}_{\text {overall }}$ of $80 \%$ at FAR1000 of 10 for the first requirement; not excluding more cows than twice the percentage as indicated by the gold standard for the second requirement). They believed farmers could make the trade-off as part of their investment decision: a less expensive sensor system may generate more false positive alerts, but this may be acceptable for some farmers. Therefore, Se levels should be presented as a range of FAR1000 levels (as done in the current paper) for the first requirement and a ranking curve, as in Figure 3, in addition to the results presented in a table for the second requirement, so that farmers can evaluate the system performance according to their own preferences.

The technology suppliers require an incentive to evaluate their systems using the proposed protocols. This incentive is likely to come from the market in the form of farmers asking for protocol-approved sensors. Eastwood et al. (2015) identified a mix of private company and industry roles in the development and support of precision dairy technologies. Protocols, such as those validated in our study, require support from both technology suppliers and the wider dairy industry in order for them to be accepted and regularly used. A good level of awareness of the protocols exists among mastitis-detection technology suppliers in New Zealand due to their involvement in the current study. However, many of the technologies have been developed and marketed in other countries and an international approach will need to be taken to refine and gain agreement on standard protocols.

\section{CONCLUSIONS}

The methodology for requirement 1 of the protocols was relatively labor intensive and required organization of personnel with reliable observational skills over an extended period and depended on mastitis incidence. The methodology for requirement 2 was shown to be straightforward to implement and only required data from an accredited milk recording test day provider in combination with the sensor data. However, no major issues were encountered during the field validation of both protocols; thus, the protocols were demonstrated to be practical. Moreover, the reported wide range of sensor performance supports the need for evaluation on at least 3 farms. The review led to the following recom- 
mended refinements to the original protocols: to opt for an analysis excluding low-density clots in addition to the analyses where all clot observations are included, regardless their density score. This will require classification of clot density for companies wishing to present these results separately. Discussion on the timeliness of alerts for $\mathrm{CM}$ led to the recommendation of extending the time window for expected $\mathrm{Se}_{\text {timely }}$ alerts from 4 to 5 cow milkings, and also setting the limit of 10 cow milkings in which a mastitis-detection system can alert for clinical mastitis for $\mathrm{Se}_{\text {overall. }}$ It is recommended that no minimum performance standards be used, and instead farmers make their own trade-offs between performance and price. These refinements of the protocols will offer a framework that allow suppliers to evaluate their technology and to present sensible information in an understandable manner to farmers. When farmers want to invest in mastitis-detection systems, they can now use the results to compare systems to support their investment decisions. The current study provides information to guide further adaptation of the protocols. Adaptation, and wider industry acceptance and use of the protocols, rely on ratification at an international level involving technology suppliers, industry organizations, and dairy farmers.

\section{ACKNOWLEDGMENTS}

The authors thank participating farmers for access to their farms, herd data, and assistance with sampling or data collection, and technology suppliers for identification of potential farms and assistance with data capture. We also thank Paul Edwards (DairyNZ Ltd., Hamilton, New Zealand) for his organization in recruiting farms and coordinating milk recording test days. This study was funded by New Zealand dairy farmers through DairyNZ Inc. in partnership with Ministry of Primary Industries (Wellington, New Zealand) through Primary Growth Partnership funding. All procedures were approved by the Ruakura Animal Ethics Committee (Hamilton, New Zealand).

\section{REFERENCES}

Borchers, M. R., and J. Bewley. 2015. An assessment of producer precision dairy farming technology use, prepurchase considerations, and usefulness. J. Dairy Sci. 98:4198-4205. http://dx.doi. org/10.3168/jds.2014-8963.

Claycomb, R. W., P. T. Johnstone, G. A. Mein, and R. A. Sherlock. 2009. An automated in-line clinical mastitis detection system using measurement of conductivity from foremilk of individual udder quarters. N. Z. Vet. J. 57:208-214.
Eastwood, C. R., J. G. Jago, J. P. Edwards, and J. K. Burke. 2015. Getting the most out of advanced farm management technologies: roles of technology suppliers and dairy industry organisations in supporting precision dairy farmers. Anim. Prod. Sci. http:// dx.doi.org/10.1071/AN141015.

Edwards, J. P., B. T. Dela Rue, and J. Jago. 2015. Evaluating rates of technology adoption and milking practices on New Zealand Dairy Farms. Anim. Prod. Sci. 55:702-709. http://dx.doi.org/10.1071/ AN14065.

Edwards, J. P., J. G. Jago, and N. Lopez-Villalobos. 2013. Large rotary dairies achieve high cow throughput but are not more labour efficient than medium sized rotaries. Anim. Prod. Sci. 53:573-579. http://dx.doi.org/10.1071/AN12312.

Hockey, C., J. M. Morton, S. T. Norman, and M. R. McGowan. 2010. Evaluation of a neck mounted 2-hourly activity meter system for detecting cows about to ovulate in two paddock-based Australian dairy herds. Reprod. Domest. Anim. 45:e107-e117. http://dx.doi. org/10.1111/j.1439-0531.2009.01531.x.

Hogeveen, H., C. Kamphuis, W. Steeneveld, and H. Mollenhorst. 2010. Sensors and clinical mastitis - The quest for the perfect alert. Sensors (Basel) 10:7991-8009. http://dx.doi.org/10.3390/s100907991.

Jago, J., C. Eastwood, K. Kerrisk, and I. Yule. 2013. Precision dairy farming in Australasia: Adoption, risks and opportunities. Anim. Prod. Sci. 53:907-916. http://dx.doi.org/10.1071/AN12330.

Kamphuis, C., B. Dela Rue, G. Mein, and J. Jago. 2013a. Development of protocols to evaluate in-line mastitis detection systems. J. Dairy Sci. 96:4047-4058. http://dx.doi.org/10.3168/jds.2012-6190.

Kamphuis, C., E. Frank, J. K. Burke, G. A. Verkerk, and J. Jago. 2013b. Applying additive logistic regression to data derived from sensors monitoring behavioral and physiological characteristics of dairy cows to detect lameness. J. Dairy Sci. 96:7043-7053. http:// dx.doi.org/10.3168/jds.2013-6993.

Kamphuis, C., H. Mollenhorst, J. A. P. Heesterbeek, and H. Hogeveen. 2010a. Data mining to detect clinical mastitis with automatic milking. Pages 568-572 in Proceedings of the 5th IDF International Mastitis Conference: Mastitis Research into Practice. J. E. Hillerton, ed. Christchurch, New Zealand. Vetlearn, Wellington, New Zealand.

Kamphuis, C., H. Mollenhorst, J. A. P. Heesterbeek, and H. Hogeveen. 2010b. Detection of clinical mastitis with sensor data from automatic milking systems is improved by using decision-tree induction. J. Dairy Sci. 93:3616-3627. http://dx.doi.org/10.3168/ jds.2010-3228.

Miekley, B., I. Traulsen, and J. Krieter. 2013. Detection of mastitis and lameness in dairy cows using wavelet analysis. Livest. Sci. 148:227-236. http://dx.doi.org/10.1016/j.livsci.2012.06.010.

Mollenhorst, H., L. J. Rijkaart, and H. Hogeveen. 2012. Mastitis alert preferences of farmers milking with automatic milking systems. J. Dairy Sci. 95:2523-2530. http://dx.doi.org/10.3168/jds.2011-4993.

New Zealand Standard. 2007. Dairy Herd Testing (NZS 8100:2007). Standards New Zealand, Wellington, New Zealand.

Russell, R. A., and J. Bewley. 2013. Characterization of Kentucky dairy producer decision-making behavior. J. Dairy Sci. 96:47514758. http://dx.doi.org/10.3168/jds.2012-6538.

Rutten, C. J., A. G. J. Velthuis, W. Steeneveld, and H. Hogeveen. 2013. Invited review: Sensors to support health management on dairy farms. J. Dairy Sci. 96:1928-1952. http://dx.doi.org/10.3168/ jds.2012-6107.

Sherlock, R., H. Hogeveen, G. Mein, and M. Rasmussen. 2008. Performance evaluation of systems for automated monitoring of udder health: Analytical issues and guidelines. Pages 275-282 in Proceedings Mastitis Control-From Science to Practice. T. J. G. M. Lam, ed. Wageningen Academic Publishers, Wageningen, the Netherlands.

Steeneveld, W., and H. Hogeveen. 2015. Characterization of Dutch dairy farms using sensor systems for cow management. J. Dairy Sci. 98:709-717. http://dx.doi.org/10.3168/jds.2014-8595. 